\title{
Avaliação da imunomarcação de Fibronectina e Tenascina induzida por cimentos biocerâmicos reparadores: estudo em tecido subcutâneo de ratos wistar
}

Evaluation of Fibronectin and Tenascin imunolabeling induced by bioceramic repair cements: study in subcutaneous tissue of wistar rats

Evaluación de la inmunotinción de Fibronectina y Tenascina inducida por la reparación de cementos biocerámicos: un estudio en tejido subcutáneo de ratas wistar

Diego Valentim

ORCID: https://orcid.org/0000-0002-5699-6578 Universidade Estadual Paulista, Brasil E-mail: dvalentim_5@hotmail.com

Carlos Roberto Emerenciano Bueno ORCID: https://orcid.org/0000-0002-1897-2823

Universidade Estadual do Norte do Paraná, Brasil E-mail: dentistabueno@gmail.com

Marina Tolomei Sandoval Cury ORCID: https://orcid.org/0000-0003-0733-7936 Universidade Estadual Paulista, Brasil E-mail: ma.tolomei@gmail.com

Ana Maria Veiga Vasques ORCID: https://orcid.org/0000-0002-1211-2363 Universidade Estadual Paulista, Brasil E-mail: anavvasques@hotmail.com

Ana Claudia Rodrigues da Silva ORCID: https://orcid.org/0000-0002-7570-6126 Universidade Estadual Paulista, Brasil E-mail: claudia.silva@unesp.br Francine Benetti ORCID: https://orcid.org/0000-0002-5459-353X

Universidade Federal de Minas Gerais, Brasil

E-mail: Francine_Benetti@hotmail.com Gustavo Sivieri-Araújo

ORCID: https://orcid.org/0000-0002-8402-7408 Universidade Estadual Paulista, Brasil

E-mail: gustavo.sivieri@unesp.br

Rogério Castilho Jacinto

ORCID: https://orcid.org/0000-0002-2362-8920 Universidade Estadual Paulista, Brasil E-mail: rogerio.castilho@unesp.br João Eduardo Gomes Filho

ORCID: https://orcid.org/0000-0001-5994-2287 Universidade Estadual Paulista, Brasil E-mail: joao.eduardo@unesp.br Edilson Ervolino

ORCID: https://orcid.org/0000-0003-4859-0583 Universidade Estadual Paulista, Brasil E-mail: e.ervolino@unesp.br

Luciano Tavares Angelo Cintra ORCID: https://orcid.org/0000-0003-2348-7846 Universidade Estadual Paulista, Brasil

E-mail: luciano.cintra@unesp.br Eloi Dezan Junior

ORCID: https://orcid.org/0000-0002-5699-6578 Universidade Estadual Paulista, Brasil E-mail: eloi.dezan@unesp.br

\section{Resumo}

O objetivo do estudo foi avaliar a presença de imunomarcadores fibronectina e tenascina em subcutâneo de rato com cimento reparador biocerâmico Biodentine ${ }^{\circledR}$, quando comparado ao MTA Branco Angelus ${ }^{\circledR}$ e $\mathrm{Ca}(\mathrm{OH})_{2}$. Foram 
implantados tubos de polietileno no subcutâneo de 32 ratos machos Wistar contendo os materiais e um tubo vazio para controle ( $\mathrm{n}=8$ animais). Após os dias 7, 15, 30 e 60, os animais foram eutanasiados, os tubos de polietileno removidos com os tecidos circundantes e os espécimes foram preparados para análise de imunomarcação. Os dados foram analisados por meio do teste de Kruskal-Wallis e Dunn com nível de significância de 5\%. Os materiais apresentaram moderado padrão de imunomarcação para fibronectina a partir de 7 dias e de tenascina a partir de 15 dias. $\mathrm{O}$ grupo Biodentine aos 60 dias foi o único que apresentou alto padrão de imunomarcação para as glicoproteínas. Conclui-se que o cimento Biodentine induziu maior padrão de imunomarcação de tenascina e fribronectina no período de 60 dias, e se igualando ao MTA e ao $\mathrm{Ca}(\mathrm{OH})_{2}$ nos outros períodos, mostrando resultados superiores aos outros materiais.

Palavras-chave: Endodontia; Imuno-histoquímica; Inflamação, Teste de materiais; Tenascina.

\section{Abstract}

The aim of the study was to evaluate the presence of fibronectin and tenascin immunomarkers in the subcutaneous tissue of rats with Biodentine ${ }^{\circledR}$ bioceramic repair cement, when compared to White Angelus $\mathrm{MTA}^{\circledR}$ and $\mathrm{Ca}(\mathrm{OH})_{2}$. Polyethylene tubes were implanted in the subcutaneous tissue of 32 male Wistar rats containing the materials and an empty tube for control ( $\mathrm{n}=8$ animals). After days 7, 15, 30 and 60, the animals were euthanized, the polyethylene tubes removed with the surrounding tissues, and specimens were prepared for immunostaining analysis. Data were analyzed using the Kruskal-Wallis and Dunn test with a significance level of 5\%. The materials showed a moderate pattern of immunostaining for fibronectin after 7 days and tenascin after 15 days. The Biodentine ${ }^{\circledR}$ group at 60 days was the only one that showed a high standard of immunostaining for glycoproteins. We can conclude that Biodentine ${ }^{\circledR}$ cement induced a higher pattern of immunostaining of tenascin and fribronectin within 60 days, and equaling White Angelus $\mathrm{MTA}^{\circledR}$ and $\mathrm{Ca}(\mathrm{OH})_{2}$ in the other periods, showing superior results to other materials.

Keywords: Endodontics; Immunohistochemistry; Inflammation; Materials testing; Tenascin.

\section{Resumen}

El objetivo del estudio fue evaluar la presencia de inmunomarcadores de fibronectina y tenascina en el tejido subcutáneo de ratas con cemento reparador biocerámico Biodentine ${ }^{\circledR}$, en comparación con MTA Blanco Angelus ${ }^{\circledR}$ y $\mathrm{Ca}(\mathrm{OH})_{2}$. Se implantaron tubos de polietileno en el tejido subcutáneo de 32 ratas Wistar macho que contenían los materiales y un tubo vacío para el control ( $\mathrm{n}=8$ animales). Después de los días 7, 15, 30 y 60, se sacrificó a los animales, se retiraron los tubos de polietileno con los tejidos circundantes y se prepararon las muestras para el análisis de inmunotinción. Los datos se analizaron mediante la prueba de Kruskal-Wallis y Dunn con un nivel de significancia del 5\%. Los materiales mostraron un patrón moderado de inmunotinción para fibronectina después de 7 días y tenascina después de 15 días. El grupo Biodentine a los 60 días fue el único que mostró un alto nivel de inmunotinción para las glicoproteínas. Podemos concluir que el cemento Biodentine indujo un mayor patrón de inmunotinción de tenascina y fribronectina en 60 días, igualando MTA Blanco Angelus ${ }^{\circledR}$ y $\mathrm{Ca}(\mathrm{OH})_{2}$ en los otros períodos, mostrando resultados superiores a otros materiales.

Palabras clave: Endodoncia; Inmunohistoquímica, Inflamación; Ensayo de materiales; Tenascina.

\section{Introdução}

As perfurações radiculares, são caracterizadas por uma comunicação entre o sistema de canais radiculares e a superfície dentária dos tecidos periapicais (American Association of Endodontists, 2016). Alguns fatores podem predispor a acidentes ou erros, por exemplo, a presença de nódulos pulpares, dentes inclinados ou rotacionados no arco, cáries extensas, reabsorções radiculares internas, canais radiculares e câmara pulpar calcificadas (Estrela et al., 2017). A sobrevida de um dente tratado endodonticamente com histórico de perfuração radicular depende dos resultados multifatoriais, sendo esses biológicos e mecânicos (Estrela et al., 2018; Holland et al., 2001).

Ao contrário dos materiais obturadores de canais radiculares, os cimentos obturadores utilizados para selar perfurações radiculares são posicionados em contato direto com os tecidos periapicais. A resposta tecidual desses materiais, portanto, torna-se essencial e pode influenciar no resultado do tratamento endodôntico (Solanki, Venkappa \& Shan, 2018).

A biocompatibilidade e capacidade de mineralização de materiais biológicos pode ser avaliada por meio de implantes subcutâneos preenchidos com os materiais a serem testados em animais (Valentim et al., 2021). As condições devem ser 
adequadas ao tempo de permanência desse material em contato com o tecido e as reações do material podem ser consideradas para a compreensão da resposta biológica. Os parâmetros avaliados são fenômenos relacionados ao infiltrado inflamatório, reparo e características dos restos do material (Bueno et al., 2016; Bueno et al., 2017; Bueno et al., 2018; Bueno et al., 2019).

A endodontia está em constantemente avanço, e diversos materiais destinados para perfurações radiculares são disponibilizados constantemente, dentre eles, cimentos a base de silicato de cálcio, também denomidados de biocerâmicos (Estrela et al., 2018). Após hidratação, os cimentos biocerâmicos, formam uma estrutura rígida mineralizada, uma vez que possuem em sua composição silicato dicálcico e tricálcico (Camilleri, 2007). A principal vantagem da utilização dos cimentos biocerâmicos são suas propriedades físico-químicas e biológicas, capacidade de selar hermeticamente, formando ponte de dentina e apresentar biocompatibilidade (Wang, 2015).

A característica de ser biocompatível está relacionada à sua semelhança com a hidroxiapatita biológica, não sendo tóxica e aumentando a secreção de TGF-B1 (fator de crescimento) das células pulpares, o que causa angiogênese, recrutamento de células progenitoras, diferenciação celular e mineralização (Singh, Kaur, Markan \& Kapoon., 2014).

No tecido pulpar, a matriz extracelular (MEC) possui uma variedade de proteínas e polissacarídeos, responsáveis pela indução da formação de uma barreira de dentina reacionária ou reparadora (Goldberg \& Smith, 2004). A fibronectina (FNC) e a tenascina (TNC) são as duas principais glicoproteínas não colágenas envolvidas no processo de cicatrização e na odontogênese, por induzirem a diferenciação de odontoblastos (Piva, Tarquínio, Damarco, Silva \& Araújo, 2006; Thesleff,, Vaahtokari \& Partanen, 1995; Zarrabi, Javidi, Jafarian Joushan, 2011).

FNCs são proteínas adesivas compostas por 2 subunidades de alto peso molecular com várias isoformas (Nakashima, 2005). São responsáveis por uma variedade de funções celulares, como adesão, migração, crescimento e diferenciação celular (Benetti et al., 2021; Piva et al., 2006). Além disso, a FNC pode estar envolvida na migração de odontoblastos (Yoshiba, Yoshiba, Iwaku, Nakamura \& Ozawa, 1994).

A TNC é uma grande glicoproteína oligomérica da MEC, secretada por fibroblastos e células gliais em culturas de tecidos, importante para a diferenciação de odontoblastos, (Piva et al., 2006; Zarrabi et al., 2011), portanto pode estar associado à formação de dentina secundária durante o processo de reparo (Benetti et al., 2021; Piva et al., 2006).

Dentre os inúmeros cimentos reaparadores biocerâmicos disponíveis no mercado, o Biodentine ${ }^{\circledR}$ (Septodont, Saint Maur des Fossés, France), comercialmente disponível em 2009, foi formulado utilizando a tecnologia do cimento à base de MTA com propriedades semelhantes à dentina (Laurent, Camps \& About, 2012), o qual consiste em pó e líquido. O pó contém silicato tricálcico e dicálcico $\left(3 \mathrm{CaO} \mathrm{SiO}_{2}\right.$ e $2 \mathrm{CaO} \mathrm{SiO}$ ), o principal componente do cimento Portland, bem como carbonato de cálcio $\left(\mathrm{CaCO}_{3}\right)$ e dióxido de zircónio $\left(\mathrm{ZrO}_{2}\right)$ servindo como meio de contraste. O líquido é composto de cloreto de cálcio $\left(\begin{array}{lll}\mathrm{CaCl}_{2} & 2 \mathrm{H}_{2} \mathrm{O}\end{array}\right)$, usado como um acelerador, numa solução aquosa com uma mistura de policarboxilato (um agente superplastificante) (Chicarelli et al., 2021; Laurent et al., 2012).

Outro material amplamente avaliado para o tratamento de perfurações nos canais radiculares é o hidróxido de cálcio, com resultados bem difundidos na literatura científica (Estrela \& Holland, 2003; Holland et al., 1999; Holland et al., 2002). A reação entre o hidróxido de cálcio e o dióxido de carbono tissular forma carbonato de cálcio, essencial para mineralização, favorecendo o processo de reparo (Bueno et al., 2016; Estrela et al., 1995). A liberação de íons cálcio estimula a síntese de fibronectina nas células da polpa dentária, induzindo a diferenciação das células da polpa dentária em células formadoras de tecido mineralizadas (Mizuno \& Banzai, 2008).

O agregado de trióxido mineral (MTA) pode ser empregado como alternativa ao $\mathrm{Ca}(\mathrm{OH})_{2}$ para tratamento de injúrias pulpares, estimulando a formação de pontes de dentina. O MTA é um material bioativo, biocompatível, estável e com alta capacidade de vedação (Bueno et al., 2019; Eskandarizadeh, Shahpasandzadeh, Shahpasandzadeh, Torabi \& Parirokh,, 2011; 
Faraco \& Holland, 2001). Entretanto, o MTA é difícil de ser manipulado, seu tempo de presa é longo, possui custos elevados e pode causar pigmentação do elemento dental (Bueno et al., 2019; Dammaschke, Gerth, Zuchner \& Schafer, 2005).

Visando a qualidade e eficácia do reparo promovido pelos materiais disponíveis, o objetivo deste estudo foi avaliar a presença de imunomarcadores FNC e TNC em subcutâneo de rato com cimento reparador biocerâmico Biodentine ${ }^{\circledR}$, quando comparado ao MTA Branco Angelus ${ }^{\circledR}$ e $\mathrm{Ca}(\mathrm{OH})_{2}$.

\section{Metodologia}

A pesquisa foi realizada após aprovação do Comitê de Ética da Faculdade de Odontologia de Araçatuba CEUAUNESP, Processo $n^{\circ}$ 2014-00452. Trinta e dois ratos Wistar machos, pesando 250-280g, foram utilizados neste estudo. Os animais foram alojados em gabinetes com temperatura controlada e com água e comida ad libitum. O cálculo amostral foi baseado em estudos prévios (Benetti et al., 2019, Benetti et al., 2021; Cintra., 2017).

\section{Implante subcutâneo}

Cento e vinte oito tubos de polietileno foram confeccionados (Abbott Laboratories do Brasil, São Paulo, SP, Brasil), com um diâmetro interno de 1,0 mm, diâmetro externo 1,6 mm, e 10,0 mm de comprimento (Benetti et al., 2020; Bueno et al., 2018; Bueno et al., 2019). Destes, 96 foram preenchidos com um dos matreriais experimentais: $\mathrm{Ca}(\mathrm{OH})_{2}+$ água destilada, MTA Angelus Branco ${ }^{\circledR}$, Biodentine ${ }^{\circledR}$ e 32 tubos foram utilizados como controle e não foram preenchidos.

Para realização da intervenção cirúrgica, 32 animais divididos em 4 períodos de tempo $(\mathrm{n}=8)$ foram submetidos à anestesia geral com xilazina $(25 \mathrm{mg} / \mathrm{kg})$ e quetamina $(50 \mathrm{mg} / \mathrm{kg})$, via intramuscular. Uma pequena área dorsal foi depilada, a antissepsia foi obtida com solução de iodo a 5\%, foi realizada uma incisão longitudinal com lâmina de bisturi número $15 \mathrm{C}$ acompanhando a linha mediana, com extensão de no máximo $1 \mathrm{~cm}$ atingindo o tecido subcutâneo. Desta forma, com o auxílio de um trocarte adaptado os tubos de polietileno foram implantados do lado direito e esquerdo no tecido subcutâneo dos animais, esses tubos continham $\mathrm{Ca}(\mathrm{OH})_{2}$ +água destilada, MTA Angelus Branco ${ }^{\circledR}$, Biodentine ${ }^{\circledR}$, e um tubo vazio como controle. A pele foi suturada com fio de seda 4-0 (Johnson \& Johnson Produtos Profissionais Ltda, São José dos Campos, Brasil).

Após 7, 15, 30 e 60 da implantação, os animais foram eutanasiados por injeção intraperitoneal de Tiopental sódico e os tubos, com os tecidos circundantes, foram removidos e fixados em solução formalina a $10 \%$ tamponada em $\mathrm{pH}$ neutro por 24 horas, seguido de lavagem em água corrente por 12 horas. Os espécimes foram incluídos em parafina para obtenção de cortes seriados de $5 \mu \mathrm{m}$ para aplicação de imunomarcadores de FNC e TNC

Para a imunomarcação, o critério para o estabelecimento dos escores foi baseado em estudo prévios (Benetti et al., 2018; Benetti et al., 2021): 0 = ausência de imunomarcação; 1 = baixo padrão de imunomarcação; 2 = moderado padrão de imunomarcação; 3 = alto padrão de imunomarcação; 4 = padrão extremamente alto de imunomarcação, sob magnificação de 400x. Os dados foram analisados estatisticamente pelo teste de Kruskal-Wallis e Dunn; $\mathrm{p}<0.05$ foi considerado significativo.

\section{Resultados}

\section{Fibronectina}

Ao se avaliar a imunomarcação da glicoproteína fibronectina, em subcutâneo, no período de 7 dias não houve diferença entre os grupos p>0,05. No período de 15 dias os materiais MTA Branco, Biodentine e $\mathrm{Ca}(\mathrm{OH})_{2}$ apresentaram diferença estatística em relação grupo controle $(\mathrm{p}<0,05)$. No período de 30 dias os grupos MTA Branco e Biodentine apresentaram mais imunomarcação, mas sem diferença com relação ao $\mathrm{Ca}(\mathrm{OH})_{2}(\mathrm{p}>0,05)$. Já no período de 60 dias o Biodentine apresentou um alto padrão de imunomarcação $\mathrm{p}<0,05$ (Tabela 1/ Figura 1). 


\section{Tenascina}

Ao se avaliar a imunomarcação da Tenascina, em subcutâneo, no período de 7 dias não houve diferença entre os grupos p $>0,05$. No período de 15, 30 e 60 dias o Biodentine apresentou mais imunomarcação em relação aos demais grupos p $<0,05$ (Tabela 2/ Figura 2).

Tabela 1. Classificação dos espécimes de acordo com escores para marcação imunohistoquímica de FNC. Letras sobrescritas iguais não demonstram diferença estatística.

\begin{tabular}{|c|c|c|c|c|c|c|}
\hline \multirow{2}{*}{ Tempo } & \multirow{2}{*}{ Material } & \multicolumn{4}{|c|}{ Escores para FNC } & \multirow{2}{*}{ Median: } \\
\hline & & 1 & 2 & 3 & 4 & \\
\hline \multirow[t]{4}{*}{7 dias } & Controle & $8 / 8$ & 0 & 0 & 0 & $1^{\mathrm{a}}$ \\
\hline & MTA Branco Angelus & $6 / 8$ & $2 / 8$ & 0 & 0 & $1^{\mathrm{a}}$ \\
\hline & Biodentine & $2 / 8$ & $6 / 8$ & 0 & 0 & $2^{\mathrm{b}}$ \\
\hline & $\mathrm{Ca}(\mathrm{OH})_{2}$ & $7 / 8$ & $1 / 8$ & 0 & 0 & $1^{\mathrm{a}}$ \\
\hline \multirow[t]{4}{*}{15 dias } & Controle & $8 / 8$ & 0 & 0 & 0 & $1^{\mathrm{a}}$ \\
\hline & MTA Branco Angelus & 0 & $6 / 8$ & $2 / 8$ & 0 & $2^{\mathrm{b}}$ \\
\hline & Biodentine & 0 & $7 / 8$ & $1 / 8$ & 0 & $2^{\mathrm{b}}$ \\
\hline & $\mathrm{Ca}(\mathrm{OH})_{2}$ & 0 & $8 / 8$ & 0 & 0 & $2^{\mathrm{b}}$ \\
\hline \multirow[t]{4}{*}{30 dias } & Controle & $8 / 8$ & 0 & 0 & 0 & $1^{\mathrm{a}}$ \\
\hline & MTA Branco Angelus & 0 & 0 & $6 / 8$ & $2 / 8$ & $3^{\mathrm{b}}$ \\
\hline & Biodentine & 0 & 0 & $7 / 8$ & $1 / 8$ & $3^{\mathrm{b}}$ \\
\hline & $\mathrm{Ca}(\mathrm{OH})_{2}$ & 0 & $6 / 8$ & $2 / 8$ & 0 & $2^{\mathrm{b}}$ \\
\hline \multirow[t]{4}{*}{60 dias } & Controle & $8 / 8$ & 0 & 0 & 0 & $1^{\mathrm{a}}$ \\
\hline & MTA Branco Angelus & 0 & 0 & $8 / 8$ & 0 & $3^{\mathrm{b}}$ \\
\hline & Biodentine & 0 & 0 & $2 / 8$ & $6 / 8$ & $4^{c}$ \\
\hline & $\mathrm{Ca}(\mathrm{OH})_{2}$ & 0 & $6 / 8$ & $2 / 8$ & 0 & $2^{\mathrm{b}}$ \\
\hline
\end{tabular}

Fonte: Autores.

Tabela 2. Classificação dos espécimes de acordo com escores para marcação imunoistoquímica de TNC. Letras sobrescritas iguais não demonstram diferença estatística.

\begin{tabular}{|c|c|c|c|c|c|c|}
\hline \multirow{2}{*}{ Tempo } & \multirow{2}{*}{ Material } & \multicolumn{4}{|c|}{ Escores para TNC } & \multirow{2}{*}{ Mediana } \\
\hline & & 1 & 2 & 3 & 4 & \\
\hline \multirow[t]{4}{*}{7 dias } & Controle & $8 / 8$ & 0 & 0 & 0 & $1^{\mathrm{a}}$ \\
\hline & MTA Branco Angelus & $8 / 8$ & 0 & 0 & 0 & $1^{\mathrm{a}}$ \\
\hline & Biodentine & $8 / 8$ & 0 & 0 & 0 & $1^{\mathrm{a}}$ \\
\hline & $\mathrm{Ca}(\mathrm{OH})_{2}$ & $8 / 8$ & 0 & 0 & 0 & $1^{\mathrm{a}}$ \\
\hline \multirow[t]{4}{*}{15 dias } & Controle & $8 / 8$ & 0 & 0 & 0 & $1^{\mathrm{a}}$ \\
\hline & MTA Branco Angelus & $7 / 8$ & $1 / 8$ & 0 & 0 & $1^{\mathrm{a}}$ \\
\hline & Biodentine & $2 / 8$ & $6 / 8$ & 0 & 0 & $2^{b}$ \\
\hline & $\mathrm{Ca}(\mathrm{OH})_{2}$ & $8 / 8$ & 0 & 0 & 0 & $1^{\mathrm{a}}$ \\
\hline \multirow[t]{4}{*}{30 dias } & Controle & $8 / 8$ & 0 & 0 & 0 & $1^{\mathrm{a}}$ \\
\hline & MTA Branco Angelus & 0 & $8 / 8$ & 0 & 0 & $2^{b}$ \\
\hline & Biodentine & 0 & $2 / 8$ & $6 / 8$ & 0 & $3^{c}$ \\
\hline & $\mathrm{Ca}(\mathrm{OH})_{2}$ & $6 / 8$ & $2 / 8$ & 0 & 0 & $1^{\mathrm{a}}$ \\
\hline \multirow[t]{4}{*}{60 dias } & Controle & $8 / 8$ & 0 & 0 & 0 & $1^{\mathrm{a}}$ \\
\hline & MTA Branco Angelus & 0 & $6 / 8$ & $2 / 8$ & 0 & $2^{b}$ \\
\hline & Biodentine & 0 & 0 & $6 / 8$ & $2 / 8$ & $3^{c}$ \\
\hline & $\mathrm{Ca}(\mathrm{OH})_{2}$ & 0 & $7 / 8$ & $1 / 8$ & 0 & $2^{b}$ \\
\hline
\end{tabular}


Fonte: Autores.

Figura 1. Grupos: Controle (A, E, I, M), Biodentine ${ }^{\circledR}(\mathrm{B}, \mathrm{F}, \mathrm{J}, \mathrm{N}), \mathrm{Ca}(\mathrm{OH})_{2}(\mathrm{C}, \mathrm{G}, \mathrm{K}, \mathrm{O})$ e MTA Angelus Branco ${ }^{\circledR}(\mathrm{D}, \mathrm{H}, \mathrm{L}$, P). Tempos de análise: 7 dias (A-D), 15 dias (E-H), 30 dias (I-L), 60 dias (M-P). [Marcação imunoistoquímica para Fibronectina; 400X].
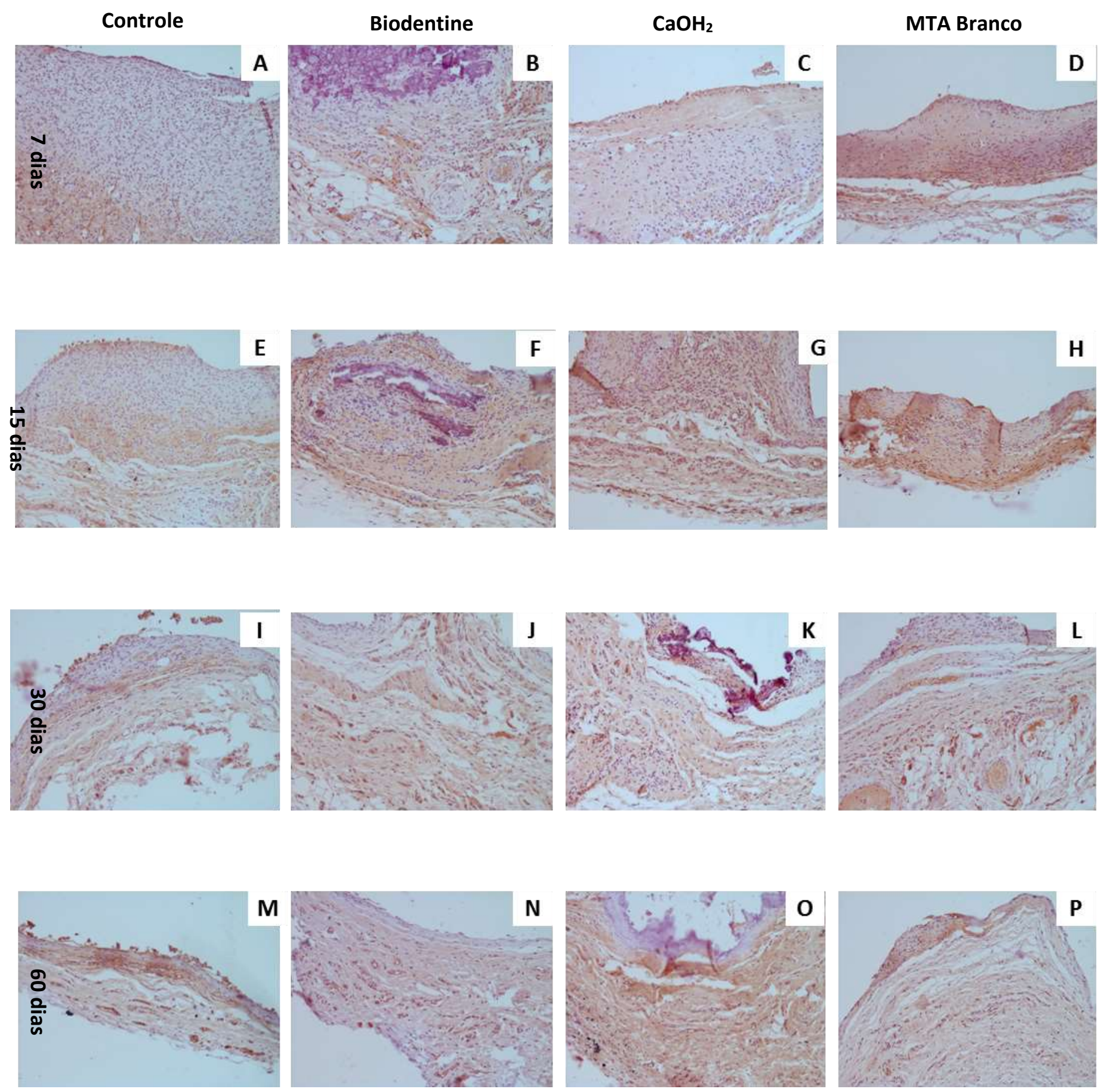

Fonte: Autores. 
Figura 2. Grupos: Controle (A, E, I, M), Biodentine ${ }^{\circledR}(\mathrm{B}, \mathrm{F}, \mathrm{J}, \mathrm{N}), \mathrm{Ca}(\mathrm{OH}) 2(\mathrm{C}, \mathrm{G}, \mathrm{K}, \mathrm{O})$ e MTA Angelus Branco ${ }^{\circledR}$ (D, H, L, P). Tempos de análise: 7 dias (A-D), 15 dias (E-H), 30 dias (I-L), 60 dias (M-P). [Marcação imunoistoquímica para Tenascina; 400X].
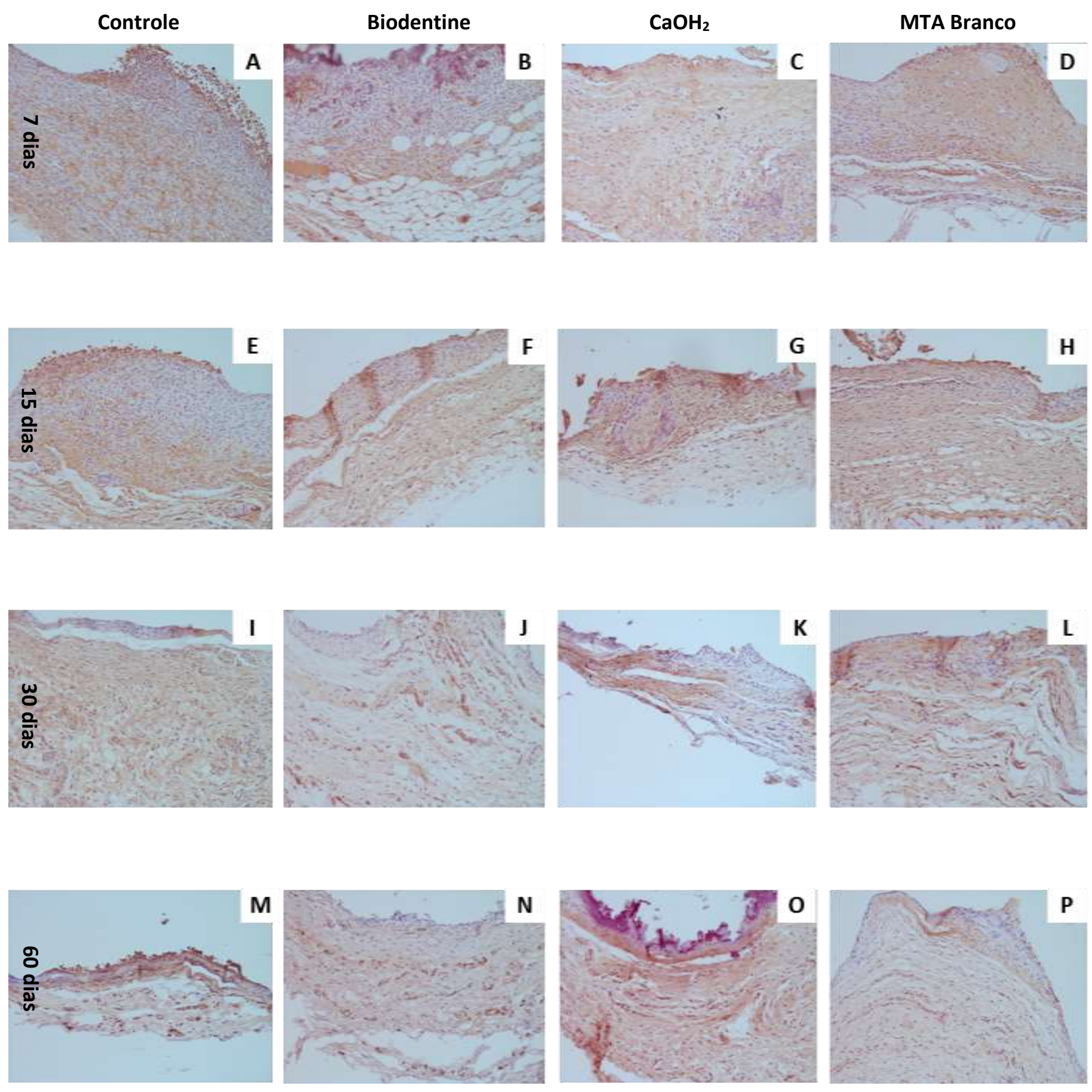

Fonte: Autores. 


\section{Discussão}

A proposta deste estudo foi avaliar a biocompatibilidade relacionada à presença das glicoproteínas Fibronectina e Tenascina dos cimentos endodônticos biocerâmicos reparadores Biodentine ${ }^{\circledR}$ e MTA Angelus Branco ${ }^{\circledR}$ comparados ao hidróxido de cálcio em subcutâneo de ratos Wistar.

A metodologia in vivo de testes em animais tem sido amplamente utilizada na endodontia, diferindo o modelo animal desde uso em cães (Dezan-Junior et al., 2021; Gomes-Filho et al., 2013, Holland et al., 2007), preás (Bhambhani \& Bolanos 1993), coelhos (Tabarsi et al., 2012) ou ratos (Bueno et al., 2021; Cintra et al., 2017).

A avaliação de compatibilidade tecidual em subcutâneo de ratos foi proposta por Torneck na década de 60 (Torneck, 1966) e tornou-se um método preliminar comumente usado para avaliar a resposta biológica a materiais (Benetti et al., 2020, Benetti et al., 2021; Bueno et al., 2016; Bueno et al., 2018; Bueno et al., 2019; Cintra et al., 2013; Valentim et al., 2021), determinada como metodologia padrão recomendada pela ISO 10993 (ISSO, 2016).

O uso de imunomarcadores como a Fibronectina e a Tenascina foi devido a função dessas duas glicoproteínas não colágenas da matriz extracelular, que está relacionada ao reparo tecidual (Chiquet-Ehrismann, 1990; Zarrabi et al., 2011). Estudos prévios avaliando materiais endodônticos utilizaram a análise imunohistoquímica de tenascina e fibronectina (Baldissera et al., 2013; Moradi et al., 2015; Zarrabi et al., 2011), embasando nossa metodologia.

Uma vez que o presente estudo usou cimentos reparadores biocerâmicos, é importante ressaltar que estudos prévios indicaram que a tenascina é necessária para a mineralização (Benetti et al., 2021; Zarrabi et al., 2011). Embora a análise subcutânea tenha sido realizada em tecido conjuntivo com ausência de células adequadas à biomineralização, como odontoblastos ou osteoblastos, a imunomarcação da tenascina indicou não apenas um processo de reparo, mas também a indução de biomineralização (Benetti et al., 2021; Piva et al., 2006).

Concomitantemente, no processo de reparo, uma matriz rica em Fibronectina pode servir como um reservatório de fatores de crescimento e moléculas que sinalizam para a diferenciação de odontoblastos em dentina terciária (Tziafas et al., 1995). Além disso, a tenascina pode facilitar a adesão entre células e a Fibronectina, facilitando a migração celular através da matriz (Zarrabi et al., 2011).

Em um recente estudo realizado para avaliar a biocompatibilidade de cimentos endodônticos biocerâmicos reparadores, Valentim et al. (2021) demonstraram que o Biodentine ${ }^{\circledR}$ induz baixa inflamação e regrediu durante o tempo experimental, quando comparado com MTA branco e hidróxido de cálcio. Este resultado corrobora resultados prévios em que o Biodentine ${ }^{\circledR}$ apresenta-se como alternativa viável (Corral Nuñez, Bosomworth, Field, Whitworth \& Valentine, 2014), com características histológicas favoráveis em relação ao hidróxido de cálcio, que antes era considerado o padrão ouro para esse tratamento. (Chicarelli et al., 2021).

$\mathrm{O}$ alto padrão de imunomarcação de fibronectina e tenascina observado neste estudo indica um processo de reparo avançado e indícios de mineralização, induzidos pelos cimentos biocerâmicos. A presença de alto padrão desses imunomarcadores relacionados ao reparo tecidual pôde ser observado em um recente estudo conduzido por Leite et al. (2021), no qual os autores sugeriram aplicação de fibronectina associada a hidrogel em polpas de dentes com rizogênese incompleta, funcionando como uma técnica de regeneração tecidual guiada e evitando um tratamento endodôntico radical.

\section{Conclusão}

De acordo com os resultados, conclui-se que cimento Biodentine induziu maior padrão de imunomarcação de tenascina e fribronectina no período de 60 dias, se igualando ao MTA e ao $\mathrm{Ca}(\mathrm{OH})_{2}$ nos outros períodos. São necessárias 
pesquisas adicionais com outros imunomarcadores, como de biomineralização e citocinas pró e anti-inflamatórias para complementar os achados desta pesquisa.

\section{Agradecimentos}

Este estudo teve apoio financeiro FAPESP, Processo 2014/02327-0.

\section{Referências}

American Association of Endodontists. (2016). Glossary of endodontic terms. 2020, in https://www.aae.org/specialty/clinical-resources/glossary-endodonticterms/.

Baldissera, E. Z., Silva, A. F., Gomes, A. P., Etges, A., Botero, T., Demarco, F. F., \& Tarquinio, S. B. (2013). Tenascin and fibronectin expression after pulp capping with different hemostatic agents: a preliminary study. Brazilian dental journal, 24(3), 188-193.

Benetti, F., Briso, A., Ferreira, L. L., Carminatti, M., Álamo, L., Ervolino, E., Dezan-Júnior, E., \& Cintra, L. (2018). In Vivo Study of the Action of a Topical Anti-Inflammatory Drug In Rat Teeth Submitted To Dental Bleaching. Brazilian dental journal, 29(6), 555-561.

Benetti, F., Bueno, C., Reis-Prado, A., Souza, M. T., Goto, J., Camargo, J., Duarte, M., Dezan-Júnior, E., Zanotto, E. D., \& Cintra, L. (2020). Biocompatibility, Biomineralization, and Maturation of Collagen by RTR $®$, Bioglass and DM Bone ${ }^{\circ}$ Materials. Brazilian dental journal, $31(5), 477-484$.

Benetti, F., Gomes-Filho, J. E., de Azevedo-Queiroz, I. O., Carminatti, M., Conti, L. C., Dos Reis-Prado, A. H., de Oliveira, S., Ervolino, E., Dezan-Júnior, E., \& Cintra, L. (2021). Biological assessment of a new ready-to-use hydraulic sealer. Restorative dentistry \& endodontics, $46(2)$, e21.

Benetti, F., Queiroz, Í., Cosme-Silva, L., Conti, L. C., Oliveira, S., \& Cintra, L. (2019). Cytotoxicity, Biocompatibility and Biomineralization of a New Readyfor-Use Bioceramic Repair Material. Brazilian dental journal, 30(4), 325-332.

Bhambhani, S. M., \& Bolanos, O. R. (1993). Tissue reactions to endodontic materials implanted in the mandibles of guinea pigs. Oral surgery, oral medicine, and oral pathology, 76(4), 493-501.

Bueno, C. R., Valentim, D., Marques, V. A., Gomes-Filho, J. E., Cintra, L. T., Jacinto, R. C., \& Dezan-Junior, E. (2016). Biocompatibility and biomineralization assessment of bioceramic-, epoxy-, and calcium hydroxide-based sealers. Brazilian oral research, $30(1)$, S1806-83242016000100267.

Bueno, C., Valentim, D., Jardim Junior, É. G., Mancuso, D. N., Sivieri-Araujo, G., Jacinto, R. C., Cintra, L., \& Dezan-Junior, E. (2018). Tissue reaction to Aroeira (Myracrodruon urundeuva) extracts associated with microorganisms: an in vivo study. Brazilian oral research, 32 , e42.

Bueno, C., Vasques, A., Cury, M., Sivieri-Araújo, G., Jacinto, R. C., Gomes-Filho, J. E., Cintra, L., \& Dezan-Júnior, E. (2019). Biocompatibility and biomineralization assessment of mineral trioxide aggregate flow. Clinical oral investigations, 23(1), 169-177.

Bueno, C.R.E., Sumida, D.H., Duarte, M.A.H., Ordinola-Zapata, R., Azuma, M.M., Guimarães, G., Pinheiro, T.N., \& Cintra, L.T.A. (2021). Accuracy of radiographic pixel linear analysis in detecting bone loss in periodontal disease: Study in diabetic rats. Saudi Dental Journal, in press.

Bueno, CRE., Lopes, GA., Valentim, D., Marques, VAS., Vasques, AMV., Cury, MTS., Dezan-Junior, E. (2017). Falhas na mistura de cimentos endodônticos: um estudo de biocompatibilidade in vivo. Brazilian Dental Science , 20 (4), 85.

Camilleri J. (2007). Hydration mechanisms of mineral trioxide aggregate. International endodontic journal, 40(6), 462-470.

Chicarelli, L., Webber, M., Amorim, J., Rangel, A., Camilotti, V., Sinhoreti, M., \& Mendonça, M. J. (2021). Effect of Tricalcium Silicate on Direct Pulp Capping: Experimental Study in Rats. European journal of dentistry, 15(1), 101-108.

Chiquet-Ehrismann, R. (1990). What distinguishes tenascin from fibrnectin. Federation of American Societes for Experimental Biology ,4, $2598-604$.

Cintra, L. T., Ribeiro, T. A., Gomes-Filho, J. E., Bernabé, P. F., Watanabe, S., Facundo, A. C., Samuel, R. O., \& Dezan-Junior, E. (2013). Biocompatibility and biomineralization assessment of a new root canal sealer and root-end filling material. Dental traumatology : official publication of International Association for Dental Traumatology, 29(2), 145-150.

Cintra, L., Benetti, F., de Azevedo Queiroz, Í. O., de Araújo Lopes, J. M., Penha de Oliveira, S. H., Sivieri Araújo, G., \& Gomes-Filho, J. E. (2017). Cytotoxicity, Biocompatibility, and Biomineralization of the New High-plasticity MTA Material. Journal of endodontics, 43(5), 774-778.

Corral Nuñez, C. M., Bosomworth, H. J., Field, C., Whitworth, J. M., \& Valentine, R. A. (2014). Biodentine and mineral trioxide aggregate induce similar cellular responses in a fibroblast cell line. Journal of endodontics, 40(3), 406-411.

Dammaschke, T., Gerth, H. U., Züchner, H., \& Schäfer, E. (2005). Chemical and physical surface and bulk material characterization of white ProRoot MTA and two Portland cements. Dental materials: official publication of the Academy of Dental Materials, 21(8), $731-738$.

Dezan-Júnior, E., Bueno, CRE., Vasques, AMV., Souza, V., de Nery, MJ., Otoboni-Filho, JA., Bernabé, PFE., Gomes-Filho, JE., Cintra, LTA., Jacinto, R., Sivieri-Araújo, G., \& Holland, R. (2021). Influence of different obturation techniques in coronal bacterial infiltration: study in dogs. Research, Society and Development, 10(4), e11010413884.

Eskandarizadeh, A., Shahpasandzadeh, M. H., Shahpasandzadeh, M., Torabi, M., \& Parirokh, M. (2011). A comparative study on dental pulp response to calcium hydroxide, white and grey mineral trioxide aggregate as pulp capping agents. Journal of conservative dentistry, 14(4), 351-355. 
Estrela, C., \& Holland, R. (2003). Calcium hydroxide: study based on scientific evidences. Journal of applied oral science : revista FOB, 11(4), 269-282.

Estrela, C., Decurcio, D. A., Rossi-Fedele, G., Silva, J. A., Guedes, O. A., \& Borges, Á. H. (2018). Root perforations: a review of diagnosis, prognosis and materials. Brazilian oral research, 32(suppl 1), e73.

Estrela, C., Pécora, J. D., Estrela, C., Guedes, O. A., Silva, B., Soares, C. J., \& Sousa-Neto, M. D. (2017). Common Operative Procedural Errors and Clinical Factors Associated with Root Canal Treatment. Brazilian dental journal, 28(2), 179-190.

Estrela, C., Sydney, G. B., Bammann, L. L., \& Felippe Júnior, O. (1995). Mechanism of action of calcium and hydroxyl ions of calcium hydroxide on tissue and bacteria. Brazilian dental journal, 6(2), 85-90.

Faraco, I. M., Jr, \& Holland, R. (2001). Response of the pulp of dogs to capping with mineral trioxide aggregate or a calcium hydroxide cement. Dental traumatology : official publication of International Association for Dental Traumatology, 17(4), 163-166.

Goldberg, M., \& Smith, A. J. (2004). Cells and extracellular matrices of dentin and pulp: a biological basis for repair and tissue engineering. Critical reviews in oral biology and medicine : an official publication of the American Association of Oral Biologists, 15(1), 13-27.

Gomes-Filho, J. E., Watanabe, S., Cintra, L. T., Nery, M. J., Dezan-Júnior, E., Queiroz, I. O., Lodi, C. S., \& Basso, M. D. (2013). Effect of MTA-based sealer on the healing of periapical lesions. Journal of applied oral science : revista FOB, 21(3), 235-242.

Holland, R., de Souza, V., Nery, M. J., Bernabé, o. F., Filho, J. A., Junior, E. D., \& Murata, S. S. (2002). Calcium salts deposition in rat connective tissue after the implantation of calcium hydroxide-containing sealers. Journal of endodontics, 28(3), 173-176.

Holland, R., de Souza, V., Nery, M. J., Otoboni Filho, J. A., Bernabé, P. F., \& Dezan Júnior, E. (1999). Reaction of rat connective tissue to implanted dentin tubes filled with mineral trioxide aggregate or calcium hydroxide. Journal of endodontics, 25(3), 161-166.

Holland, R., Filho, J. A., de Souza, V., Nery, M. J., Bernabé, P. F., \& Junior, E. D. (2001). Mineral trioxide aggregate repair of lateral root perforations. Journal of endodontics, 27(4), 281-284.

Holland, R., Mazuqueli, L., de Souza, V., Murata, S. S., Dezan Júnior, E., \& Suzuki, P. (2007). Influence of the type of vehicle and limit of obturation on apical and periapical tissue response in dogs' teeth after root canal filling with mineral trioxide aggregate. Journal of endodontics, 33(6), 693-697.

ISO - International Organization for Standardization. (2016). ISO 10993-6: Biological Evaluation of Medical Devices Part 6: Testes for Local Effects after Implantation. https://committee.iso.org/standard/61089.html?browse=tc.

Laurent, P., Camps, J., \& About, I. (2012). Biodentine(TM) induces TGF- $\beta 1$ release from human pulp cells and early dental pulp mineralization. International endodontic journal, 45(5), 439-448.

Leite, M. L., Soares, D. G., Anovazzi, G., Anselmi, C., Hebling, J., \& de Souza Costa, C. A. (2021). Fibronectin-loaded Collagen/Gelatin Hydrogel Is a Potent Signaling Biomaterial for Dental Pulp Regeneration. Journal of endodontics, 47(7), 1110-1117.

Mizuno, M., \& Banzai, Y. (2008). Calcium ion release from calcium hydroxide stimulated fibronectin gene expression in dental pulp cells and the differentiation of dental pulp cells to mineralized tissue forming cells by fibronectin. International endodontic journal, 41(11), 933-938.

Moradi, S., Saghravanian, N., Moushekhian, S., Fatemi, S., \& Forghani, M. (2015). Immunohistochemical Evaluation of Fibronectin and Tenascin Following Direct Pulp Capping with Mineral Trioxide Aggregate, Platelet-Rich Plasma and Propolis in Dogs' Teeth. Iranian endodontic journal, 10(3), $188-192$.

Nakashima M. (2005). Bone morphogenetic proteins in dentin regeneration for potential use in endodontic therapy. Cytokine \& growth factor reviews, 16(3), 369-376.

Piva, E., Tarquínio, S B., Demarco, F F., Silva, A F., \& De Araújo, V C. (2006). Immunohistochemical expression of bronectin and tenascin a er direct pulp capping with calcium hydroxide. Oral surgery, oral medicine, oral pathology, oral radiology, and endodontology,102, e66-e 71.

Singh,H., Kaur, M., Markan, S., \& Kapoor, P. (2014). Biodentine: A promising dentin substitute. Journal of Interdisciplinary Medicine and Dental Science, 2 , 140 .

Solanki, N. P., Venkappa, K. K., \& Shah, N. C. (2018). Biocompatibility and sealing ability of mineral trioxide aggregate and biodentine as root-end filling material: A systematic review. Journal of conservative dentistry : JCD, 21(1), 10-15.

Tabarsi, B., Pourghasem, M., Moghaddamnia, A., Shokravi, M., Ehsani, M., Ahmadyar, M., \& Asgary, S. (2012). Comparison of skin test reactivity of two endodontic biomaterials in rabbits. Pakistan journal of biological sciences : PJBS, 15(5), 250-254.

Thesleff, I., Vaahtokari, A., \& Partanen, A. M. (1995). Regulation of organogenesis. Common molecular mechanisms regulating the development of teeth and other organs. The International journal of developmental biology, 39(1), 35-50.

Torneck, CD. (1966). Reaction of rat connective tissue to polyethylene tube implants part I. Cirurgia Oral, Medicina Oral, Patologia Oral, 21 (3), 379-87.

Tziafas, D., Alvanou, A., Panagiotakopoulos, N., Smith, A. J., Lesot, H., Komnenou, A., \& Ruch, J. V. (1995). Induction of odontoblast-like cell differentiation in dog dental pulps after in vivo implantation of dentine matrix components. Archives of oral biology, 40(10), 883-893.

Valentim, D., Bueno, CRE., Marques, VAS., Benetti,F., Vasques, AMV., Cury, MTS., Silva, ACR., Jacinto, RC., Sivieri-Araujo, G., Cintra, LTA., \& DezanJunior, E. (2021). Biocompatibility assessment of bioceramic repair cements: An in vivo study in wistar rats. Research, Society and Development, 10(7), e1610714422. 
Research, Society and Development, v. 10, n. 10, e589101019325, 2021

(CC BY 4.0) | ISSN 2525-3409 | DOI: http://dx.doi.org/10.33448/rsd-v10i10.19325

Wang Z. (2015). Bioceramic materials in endodontics. Endodontic Topics, 32(1), 3-30.

Yoshiba, N., Yoshiba, K., Iwaku, M., Nakamura, H., \& Ozawa, H. (1994). A confocal laser scanning microscopic study of the immunofluorescent localization of fibronectin in the odontoblast layer of human teeth. Archives of oral biology, 39(5), 395-400.

Zarrabi, M. H., Javidi, M., Jafarian, A. H., \& Joushan, B. (2011). Immunohistochemical expression of fibronectin and tenascin in human tooth pulp capped with mineral trioxide aggregate and a novel endodontic cement. Journal of endodontics, 37(12), 1613-1618. 